\title{
Automatization of a multipurpose astronomical spectrograph
}

\author{
J. Sanchez del Rio and A. Ruiz-Falco Rojas \\ Instituto de Astrofisica de Andalucia, C.S.I.C., Granada, Spain \\ G. Herpe \\ Observatoire de Paris-Meudon, EROES du LAEC du CNRS, Meudon, France
}

(Received 3 March 1999; accepted for publication 14 January 2000)

\begin{abstract}
This article deals with the study and fabrication of a system for the automation of a multipurpose spectrograph used in Astronomy. This system allows the 19 movements of the instrument to run by using high-level software, a "master" board, and 19 "slave" boards. The Astrophysique Lineal et BIdimensional par RÉseaux Optiques (ALBIREO) spectrograph has three working modes and is installed in the Observatorio de Sierra Nevada, Granada, Spain. We describe a low-cost system realized for the automatization of a multifunction spectrograph. It allows the control of a large number of motors: 19 for Albireo; the theoretical limit is 32768! Another advantage of this architecture based on "slave" boards is to supply each motor with exactly the current it needs to reach the required torque (reliability and repetitivity of motions are obtained when the motor torque is at least twice the resistant torque). Thus, we can avoid unnecessary overheating which would be detrimental to the material as well as to the local image quality (seeing). The reliability of the system is obtained by software filtering the commands and also by using optocoupled electronics to avoid interference. For a complex instrument, the high-level software must be user friendly. The efficiency of the present software in this respect has been appreciated since first being used on the telescope. This system may be of interest to laboratories and observatories with similar needs for automation of motions. The adoption of this system will be supported by the IAA Laboratory, Granada, Spain. (C) 2000 American Institute of Physics. [S0034-6748(00)01606-3]
\end{abstract}

\section{INTRODUCTION}

A multipurpose spectrograph ALBIREO has been built jointly by the EROES team of the Laboratoire d'Astronomie Extragalactique et de Cosmologie in Meudon Observatory (France) and the Instituto de Astrofisica de Andalucia in Granada (Spain).

This instrument is permanently mounted at one of the two Nasmyth foci $(F / 8$, scale: 1 in. $=58.72 \mu \mathrm{m})$ of the new $1.52 \mathrm{~m}$ telescope in the Sierra Nevada Observatory (Spain) and will be used for various stellar and extragalactic programs. It provides several modes of observation, using either a long slit for classical spectroscopy or optical fibre devices for multiobject (Medusa) as well as integral field (Argus) spectroscopy.

The aim of the present article is to briefly describe the instrument and to place emphasis on the electronic and software solutions found for its automation. The latter is done through the use of high-level software for running a "master" board, itself controlling some 19 "slave" boards. Each "slave" board corresponds to the motion of a step motor. Software, "master" and "slave" boards have been developed, economically at the IAA Laboratory.

\section{ORGANIZATION OF THE INSTRUMENT}

The instrumental chain includes a mechanical interface, the spectrograph "stricto sensu," and the detector. The spectrograph itself consists of three parts: the "bonnette," the spatial stage, and the spectrographic stage.
The "rotator" is an interface between the telescope and the spectrograph that ensures a reliable and reproducible position during rotation. This is important for the multiobject mode and may be needed for the long-slit mode when a given orientation is required.

The "bonnette" fulfils the functions of field acquisition, guiding, and calibration. It contains a high-sensitivity video camera ${ }^{1}$ movable on $x, y, z$ axes. When checking the centering in the long-slit and Argus mode, a relay optics views a 18 $\mathrm{mm}(=5 \mathrm{ft})$ field around the slit or the fiber bundle entrance. This configuration may be used for guiding when a suitable (mag 18) star is found. Another configuration allows the scramming of a larger field (a ring of $5 \mathrm{ft}$ internal diameter and $20 \mathrm{ft}$ external diameter). The bonnette contains also a wavelength calibration unit with four lamps.

The "spatial stage" selects a combination of three spectrographic modes with different spatial coverage: a classical long slit of $6 \mathrm{ft}$ length and adjustable width; a multiobject spectroscopy unit (Medusa) with 40 optical fibers of $200 \mu \mathrm{m}$ (=3.4 in.) diameter plugged in an aperture plate covering 20 $\mathrm{ft}$; integral field spectroscopy (Argus) with a bundle of 202 fibers of $88 \mu \mathrm{m}(=1.5 \mathrm{in}$.) diameter covering a $16 \times 30 \mathrm{in}$. area. A fourth mode is direct imaging. All four "spatial" devices are mounted on the same sliding plate and the configuration is rapidly changed $(1 \mathrm{~min})$ by remote control. This part contains also a filter wheel with $U, B, V, R$ filters.

The "spectrograph stage" is of classical design, with a dioptric collimator $(F=336 \mathrm{~mm}, F / D=5.6)$ and camera 




FIG. 1. Principle of the spectrograph.

$(F=121 \mathrm{~mm}, F / D=2)$ and a $60 \mathrm{~mm}$ pupil. The reflection gratings give dispersions between, roughly, 200-40 A/mm giving resolved elements between 10 and $2 \mathrm{~A}$ in Argus mode. The useful wavelength range is $3500-10000 \mathrm{~A}$.

The present "detector" is a charge coupled device (CCD) integrated at IAA, based on the EEV8821/T chip with $1152 \times 770$ pixels of $22.5 \mu \mathrm{m}$. It is used with its largest dimension along the direction of dispersion. The "three-level polysilicon" technology gives a minimum readout noise in the slow reading condition at $140 \mathrm{~K}$. It is supplied with a phosphor coating to enhance the blue and UV response. The chip is front illuminated, without antiblooming channel, with high efficiency transfer charge and equipped with a 16-bit A/D converter. The gain is 10.6 electrons/step in the ADC. The read noise is 4.8 electrons rms and the saturation of a pixel is approximately 300000 electrons. The CCD is read directly by the host via a 10 Mbauds high speed link. A full frame fast read-out mode as well as an adjustable subraster are available.

Finally, another facility completes this instrument set: a unit for the preparation of aperture plates (Medusa mode) from POSS plates or prints, which is located at IAA in Granada. (Figure 1 shows the principle of the spectrograph.)

\section{ELECTRONICS OF “ALBIREO”}

\section{A. Principle of design}

Being multipurpose, "Albireo" is a complex instrument, with several configurations implying automated mechanical motions, like other astronomical instruments NORRIS, ${ }^{2}$ SILFED, ${ }^{3}$ EMMI, ... ${ }^{4}$ For designing the electronics, we thus had to take into account the following constraints:



FIG. 2. Principle of the electronics.

(1) The number of motors is large (19; one for each motion).

(2) The motions must be accurate and reproducible, and they should be independent.

(3) It would be an advantage to add or replace easily the motors.

(4) The boards must be reliable and easy for maintenance or repairing "on site."

(5) The remote control of all motors should be provided by a general controller.

(6) "Albireo" will work at high altitude $(2860 \mathrm{~m})$ environment, which could mean severe atmospheric parameters: temperature, humidity, static electricity, weak ground protection.

For these reasons, the electronics for motors control is based on a backplane bus with a "master" board connected to 19 "slave" boards (one "slave" per motor). The "master" is equipped with a PIC16C71 microcontroller" and must receive commands from the host controller by RS232C and translate it for the "slaves" via a $\mathrm{I}^{2} \mathrm{C}$ bus. The "slaves" are equipped with a PIC16C57 microcontroller" and each "slave" contains all the control functions for one motor.

Both RISC microcontrollers use HARVARD architecture, in which program and data are accessed from separate memories. (This concept allows high speed with overlapping instruction fetch and execution cycles.) These cycles are running at $15 \mathrm{MHz}$. This system can be seen in Fig. 2.

\section{B. Choice of motors and zero-point system}

Each motion, associated with a function of "Albireo," is powered by a step motor with a reduction gear. The motor torque value is twice the resistant torque value for the most unfavorable torque situation. This is adjusted for each motor. When needed, there is an antibacklash system on the mechanical axis.

The precision of each motion depends upon that of the limit switch. It was found necessary to use a detector with a low hysteresis and with high reproducibility. The choice of detector is piezoelectric with a saphir point that gives an accuracy of $\pm 1 \mu \mathrm{m}$. The type of motor is a bipolar stepper with low permanent magnet rotor inertia. 


\section{Choice of motor driver controllers}

Because the motors are powerful, the electronics must be able to supply sufficient power to overcome the maximum resistant torque (which varies with the position of the telescope).

The driver is based on a modified bipolar chopper. The choice is the L297 and L298 pair of integrated circuits. ${ }^{6}$ The L297 circuit works as a generator of phases and a chopper current controller; it has pulse and direction inputs from the PIC16C57 microcontroller. The L298 is a full-bridge transistor driver which is able to power the two motor coils with two amperes maximum current.

The modification implemented is to vary the reference voltage level in the L297 by two methods. The first is logic based on the L298 inhibit lines, the second is supplied by the PIC16C57 microcontroller.

\section{Resolution of the motor control board}

Each motor is controlled by an independent "slave", board. All boards are similar and are designed specially for this application. The microcontroller PIC16C57 is a low cost, easily programmable, UV erasable device. The command from the "master" is received by all the "slaves" at the same time through the $\mathrm{I}^{2} \mathrm{C}$ bus. But "master" command is only executed if the address is the same as that of one of the "slaves." The physical address of each board is determined by the physical position in the backpanel bus.

The functions of each board are:

(1) To listen to the host, to operate the motor driver, to read the state of the limit switch.

(2) The watch-dog hardware disables the motor power, for an error condition.

The error conditions are indicated by two light-emitting (LED) diodes on the front panel, and indicated to the "master' (and host) via software.

The low level software for the "slave" board is running in the closed-loop mode without any external interruption. The basic subroutines are:

(1) To init the motors to a parking position.

(2) To init the maximum number of steps for each motor.

(3) To move the motor to the desired position at an accelerated speed, then at constant speed, and finally, at decelerated speed.

(4) To report to the "master' (and host) the number of steps or motor position and its status.

The possible maximum step number of each motor is $2^{24}$ ( 3 bytes of 8 bits). The maximum speed is 18000 steps/s. In order to minimize the electronic noise made in the chopper process, all the chopper driver frequencies are synchronized at $82000 \mathrm{~Hz}$. The electronic box is located remotely to reduce the heat produced as this can affect the telescope image quality (seeing). The heat produced by the power driver is absorbed by a copper heat sink with sufficient dissipation. (Figure 3 shows the "slave"' board diagram.)

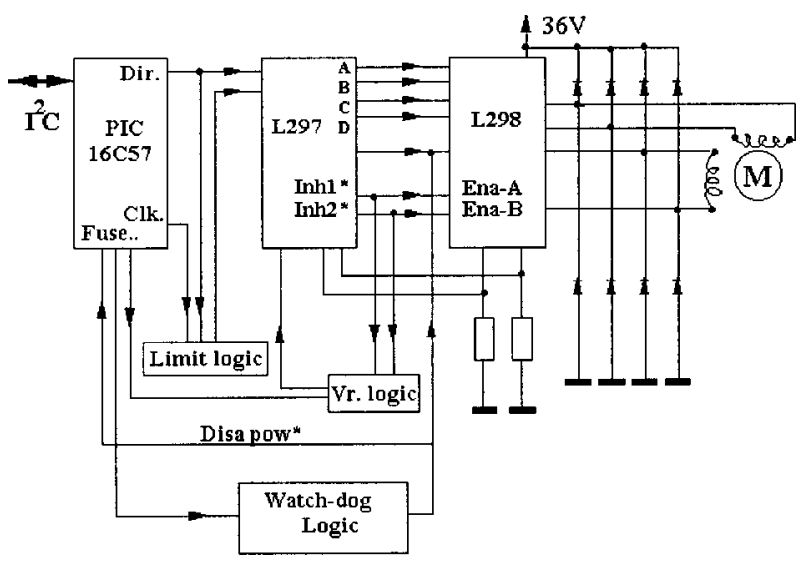

FIG. 3. Slave board diagram.

\section{E. Principle of the "master" board interface}

The instrument recognizes the received commands via RS232C from one host, checks validity and executes it. The board interface is based on PIC16C71 microcontroller, of the same family as that of the "slaves," but with an internal timer useful for the asynchronous reception of RS232C.

The received and validated commands for the motors are translated to the $\mathrm{I}^{2} \mathrm{C}$ bus, with the primary address contained in the commands. The "master" can translate a command to ask for the specified address at a "slave" board. If the motor is free, it returns its status and position, otherwise, the motor indicates busy.

The logic limit to the number of primary addresses in the $\mathrm{I}^{2} \mathrm{C}$ bus is 128 . But, by using primary and secondary addresses, this number is raised to 32768 possible motors addresses.

There is one supplementary security line to communicate to "master"' (and host) all of the error conditions. The master also controls the calibration lamps and the power line of the motors. (Figure 4 shows the "master" board diagram.)

\section{THE USER'S INTERFACE}

\section{A. Definition of the needs}

A complex instrument such as this spectrograph needs a high level of control software for extracting the data with the



FIG. 4. Master board diagram. 
maximum efficiency. Six conditions define this system:

(1) To use "Object Oriented Programming'" for a better software efficiency.

(2) To be able to run on present and future computers because the expected instrument life is larger than the present expected computer life.

(3) To display graphically all the instrument functions: status, memories,... All the controls of the instrument should be operated by mouse menu, without using complex syntaxis commands.

(4) To interact with other instruments and systems. The spectrograph belongs to a set of systems for astronomical observations and must communicate with these.

(5) To act on mechanical faults. The program must report to the user.

(6) To act on logic errors. The program must report and/or rectify the error. For example, when one is trying to take a spectrum without grism or with a wrong grism.

\section{B. The adopted solutions for the high-level software}

It is necessary to have a portable program, directly supported by Unix, Windows95, or WindowsNT operating systems, using the specific graphic interface of these systems, while maintaining a single graphic interface for the user. The following are solutions to these problems:

(1) To use $\mathrm{C}^{++}$for high level software and GNU compiler. The low level drivers are in $\mathrm{C}$ programming with basic standard compiling, as the extension compiler could give trouble with other computers.

(2) To use wx Windows graphic library for the programming, as this high-level object library is compatible with $\mathrm{C}^{++}$programming. Then Motif, Xview, and Microsoft windows software can be used.

It is essential to supply the astronomer with various functions:

(1) The status of the instrument must been permanently available, in a clearly legible graphical form. This means that a sketch of the configuration is displayed, including the actual optical path. All the instrument functions may be controlled with the mouse operating on window menus.

(2) The "Albireo" control software belongs to PICO (Programa Interactivo de COntrol) that controls the telescope, the autoguiding system, the CCD detector, "Albireo", electronics,... (see Figs. 5 and 6). Each one is a different program and works with different computers in parallel.

(3) The real-time control program must supervise all elements, with a watch-dog for each interface user, to ensure correct operation. The watch-dog sends a warning to the astronomer when an error condition is detected (for example, default communication).

(4) The reliability of the system is obtained first by filtering the commands sent by the astronomer and then by comparison between the successful commands and the instrument truth-table. For example, trying to obtain a spectrum on the sky with live calibration lamps is rejected by the truth table, as the calibration lamps must be switched off.

\section{Control Espectrografo ALBIREO}

Fichero Espectrografo Configuracion Ayuda

Rotador: $090^{\circ}$
Optica TV: sin Barlow
Espejo Cauto: origen Espejo Retomo: origen
Foco TV: $131.2 \mathrm{~mm} \quad$ Altura TV: $32.60 \mathrm{~mm}$
Transwersal TV: $27.45 \mathrm{~mm}$

Modo: Argus

Anchura Rendija: 001.5 "

Obturador: cerracto

Filtro: vacio

Lantpara: $\mathrm{Hg}$

Colimador: $-2.56 \mathrm{~mm}$

Torre Redes: red $1800 \mathrm{~V} / \mathrm{mm}$

Red 1800: $30.12^{\circ} \quad$ Red 1200R: origen
Red 1200B: origen $\quad$ Red 600: origeti

Red 300: origerl

Objetrio CCD: $\quad+1.56 \mathrm{~mm}$

Detector CCD $1152 \times 770$ pixeles:

- Time: 1200 sec. - Gain: 1

- Start: 00:00:00.0 28-DEC-98

- File: imagen12.FITS

FIG. 5. Actual display of main controls.

\section{LABORATORY AND TELESCOPE TESTING}

The spectrograph has been tested in the laboratory and on the telescope, with the following results:

(1) The resolution of the position of the moving parts is better than $0.01 \mathrm{~mm}$, taking into account all of the different errors (limit-switch reference, gauge of screws, step position errors,...).

(2) The tests of motor motions shows that the slide system functions well. A motor torque of double the resistante torque ensures that no steps are lost.

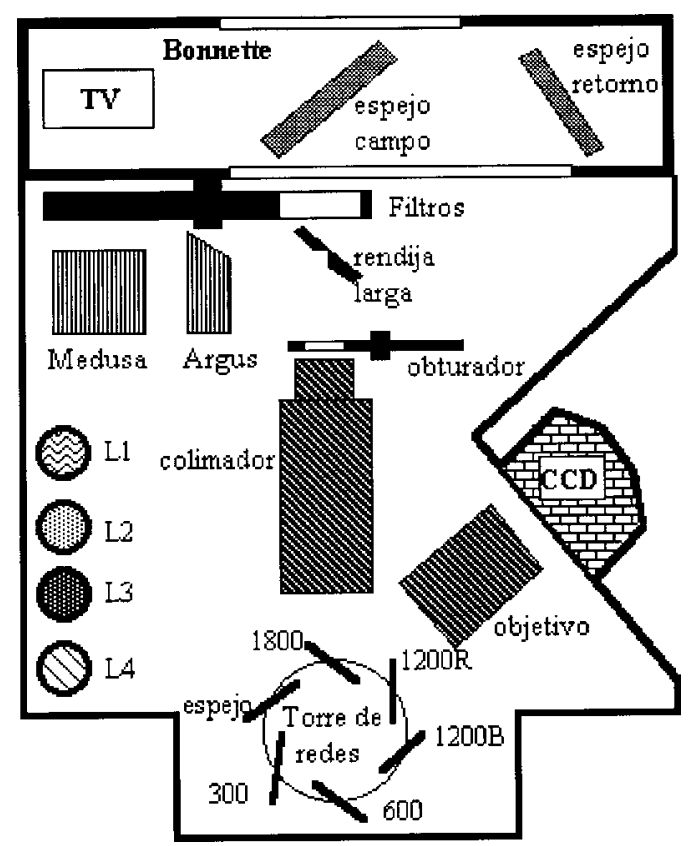

FIG. 6. Actual display of spectrograph status. 


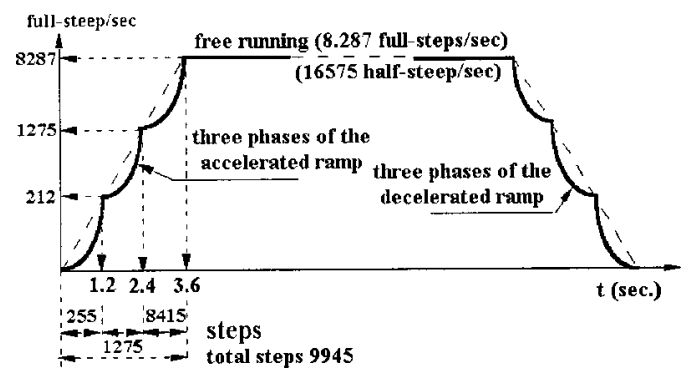

FIG. 7. Motor ramp diagram.

(3) The ambient temperature of the electronics and the instrument is between -20 and $30^{\circ} \mathrm{C}$.

(4) large motions are composed of three successive phases: First, an acceleration ramp, then a period running at maximum speed ending with a deceleration ramp. The slope of ramps is 16575 half steps during $3.6 \mathrm{~s}$. The running period is 16575 half steps/s. The program also allows the motors to run for a small number of steps with only an acceleration and a deceleration ramp (short distance).

(5) To emulate an accelerated and decelerated motion, a three phase variable speed is adopted. This involves no hard mathematical low-level subroutines. It is necessary then to adhere to the maximum step to step acceleration rate $(\mathrm{rad} / \mathrm{s})$ specified in the data sheet of the motor (see Fig. 7).

(6) The output current through the motor coils is ad- justed by a variable resistor on the "slave" board. The current, proportional to the torque, can be between 0.2 and 3.8 A per phase.

(7) The $90 \times 90 \times 10 \mathrm{~mm}$ heat absorber radiator is sufficient for the power driver when the working time is less than $25 \%$.

\section{ACKNOWLEDGMENTS}

The authors warmly thank M. Atienza, J. R. Fremy, and S. Garcia-Creus for technical help. They also thank the Observatorio de la Sierra Nevada and its team which allowed them to carry out technical tests using their telescope. They are grateful to As. del Olmo and Ch. Vanderriest for helpful, judicious advice and their collaboration on the observations at the OSN. The development was financially supported by the Instituto de Astrofisica de Andalucia, from the CSIC, Granada, Spain.

${ }^{1}$ G. Herpe, M. Chevreton, J. C. Robin, P. Prat, B. Servan, and F. Gex, Exp. Astron. 2, 163 (1991).

${ }^{2}$ D. Hamilton, J. B. Oke, M. A. Carr, F. H. Harris, J. Cohen, E. Emery, and L. Blakeé, Publ. Astron. Soc. Pac. 105, 1308 (1993).

${ }^{3}$ C. Vanderriest, Astron. Soc. Pac. Conf. Ser. 37, 338 (1993).

${ }^{4}$ S. D'odorico, Messenger 61, 51 (1990).

${ }^{5}$ PIC 16/17 Microcontroller Data Book 1995/1996, MICROCHIP, pp. 2-95 and 2-517.

${ }^{6}$ L297 and L298 Motion Control Application Manual, January 1987, SGS, pp. $443-458$. 